This item was submitted to Loughborough's Research Repository by the author.

Items in Figshare are protected by copyright, with all rights reserved, unless otherwise indicated.

\title{
Printed hydrogel nanocomposites: fine-tuning nanostructure for anisotropic mechanical and conductive properties
}

PLEASE CITE THE PUBLISHED VERSION

https://doi.org/10.1007/s42114-020-00161-5

\section{PUBLISHER}

Springer

VERSION

AM (Accepted Manuscript)

\section{PUBLISHER STATEMENT}

This is a post-peer-review, pre-copyedit version of an article published in Advanced Composites and Hybrid Materials. The final authenticated version is available online at: https://doi.org/10.1007/s42114-020-00161-5

\section{LICENCE}

CC BY-NC-ND 4.0

\section{REPOSITORY RECORD}

Zhao, Weiwei, Lijin Chen, Sanming Hu, Zhijun Shi, Xing Gao, and Vadim Silberschmidt. 2020. "Printed Hydrogel Nanocomposites: Fine-tuning Nanostructure for Anisotropic Mechanical and Conductive Properties". Loughborough University. https://hdl.handle.net/2134/13242422.v1. 


\title{
Printed hydrogel nanocomposites: fine-tuning nanostructure for anisotropic mechanical and conductive properties
}

\author{
Weiwei Zhao ${ }^{1}$, Lijin Chen ${ }^{1}$, Sanming Hu${ }^{2}$, Zhijun Shi ${ }^{2}$, Xing Gao ${ }^{3}$, Vadim V. Silberschmidt ${ }^{4}$
}

\begin{abstract}
Additive manufacturing of composites offers a potential for a new level of control over a material's structure at the microscale. The focus of this work is a 2-hydroxyethyl methacrylate (HEMA)-based gelation system with orderly distributed carbon nanotubes (CNTs). CNTs undergo shear-induced alignment during printing process, and retain their orientation after the polymerisation of HEMA monomers, thereby, forming a nanocomposite with anisotropic mechanical and electrical properties. It is characterised with an intensive programme of mechanical tests including quasistatic uniaxial stretching, and dynamic cyclic loadings, as well as its four-terminal sensing of conductive characteristics. A coupling effect of mechanical and electrical properties is also studied. The experimental findings are discussed in detail and demonstrate that the orientation of CNTs affects both the mechanical and electrical conductive properties of the nanocomposites in terms of its ultimate strength, resistivity, and a piezoresistive coefficient. Understanding of anisotropic electromechanical properties of printed PHEMA-CNT hydrogel nanocomposite will ultimately underpin the development of smart soft materials for diverse applications, such as biomimetic nucleus pulposus or flexible electronics.
\end{abstract}

Keywords Printed hydrogel nanocomposite $\cdot$ Anisotropic electromechanical properties $\cdot$ Nanofibre alignment $\cdot$ Carbon nanotubes

\section{Introduction}

High water-locking capacity, excellent biocompatibility and soft mechanical properties endow hydrogels with a great potential for biomedical applications, e.g. scaffolds in tissue engineering $[1,2]$, substrates for cell culturing [3,4], wound healing

Weiwei Zhao

wzhao@whut.edu.cn

1 School of Mechanical and Electronic Engineering, Wuhan University of Technology, Wuhan 430070, China

2 College of Life Science and Technology, Huazhong University of Science and Technology, Wuhan 430074, China

3 Research Centre for Medical Robotics and Minimally Invasive Surgical Devices, Chinese Academy of Sciences, Shenzhen Institutes of Advanced Technology, Shenzhen 518055, China

4 Wolfson School of Mechanical, Electrical and Manufacturing Engineering, Loughborough University, Loughborough, Leicestershire LE11 3TU, UK
[5, 6] or medical electrodes [7]. Additive manufacturing techniques also known as $3 \mathrm{D}$ printing enable a precise control of complex internal structures and the constructing routes by employing their benefits of computer aid design and freeform fabrication $[8,9]$. Introduction of $3 \mathrm{D}$ printing techniques in building of complex architectures of hydrogels resulted in successful production of useful and complex biostructures, such as multivascular networks [10] and organs [11]. Grigoryan et al. [10] developed bioinspired alveoli, capable to pump oxygen to the surrounding vascular network using 3D printing based on stereolithography. Hydrogel-based architectures with complex and controllable shape changes were reported by Wang et al. [12]. By engraving grooves on the surface of a sample with digital projection lithography, bending or twisting deformations due to internal swelling resistance of the hydrogel can be realised. Jiang et al. [1] and Wang et al. [13] state in their papers that direct ink writing (DIW) is much more suitable for fabricating synthetic hydrogels because it can print multiple gel-like materials simultaneously. The DIW technique (also called robocasting) requires that the "ink" is high viscosity liquid, like slurry, before its exit from a nozzle. After exiting from the nozzle, a curing or polymerising reaction should take place immediately to retain the shape of the exiting "ink", thereby, 
leaving a trace of the printed material. Thanks to their injectability and controllability, photocuring hydrogels are promising materials for direct printable inks.

As a result of a laminar flow inside a guiding tube system, the "ink" experiences laminated hydrodynamic shear during the whole process of transportation. So, if the "ink" incorporates rod or fibril-like nanofillers, such hydrodynamic shear is able to align them as the ink flows through the deposition nozzle $[14,15]$ (see Fig. 1a). Based on this phenomenon, a biomimetic hydrogel composite with anisotropic stiffness was developed by Gladman et al. [16]. In their work, cellulose fibrils acting as the nanofillers underwent shear-induced alignment during the DIW process, and retained their orientations inside the composite after polymerisation, thus, resulting in anisotropic mechanical properties of the composite.

In this work, a DIW-based printing technique, with the photocuring ink, primarily consisting of 2-hydroxyethyl methacrylate (HEMA), ethyleneglycol dimethacrylate (EGDMA), tetramethylethylenediamine (TEMED), polyvinylpyrrolidone (PVP), and carbon nanotubes (CNTs), was employed to build hydrogel nanocomposites with anisotropic mechanical and conductive properties resulting from the shear-induced alignment of the CNTs. Statistical analysis on the directive orientations of the CNTs (see Fig. 1d) demonstrates that over 50\% of CNTs' directive orientations fall in the offset range of $\pm 10^{\circ}$ from the aligning direction, thereby, experimentally verifies the anisotropy of the CNTs in the produced hydrogel nanocomposite. Both the mechanical and electrical properties of the printed material samples, including mechanical characteristics under conditions of quasistatic uniaxial tension and dynamic cyclic loading, as well as electric conductivity and piezoresistivity, are evaluated in this paper. A fraction of CNTs embedded in the hydrogel nanocomposite, an angle between the preferential orientation of CNTs and a loading direction of the sample and a stretching speed (strain rate) are taken as independent variables and intensively investigated to assess the anisotropic mechanical and electrical characteristics. The data presented in this work provides a fundamental understanding of both the CNT-based fine-tuning techniques and anisotropic properties of the printed hydrogel nanocomposite.

\section{Materials and methods}

\subsection{Materials}

67.5 vol\% of the monomer HEMA (purity $\geq 99 \%$, from Sigma-Aldrich Co. Ltd.), 30 vol\% of the solvent deionised water, 0.8 vol\% of the crosslinker EGDMA (purity $98 \%$, from Sigma-Aldrich Co. Ltd.) and 1.7 vol\% of the catalyser TEMED (purity $\geq 99.5 \%$, from Sigma-Aldrich Co. Ltd.) were blended to form a homogeneous aqueous solution. $10 \mathrm{w} / v \%$ of stabiliser PVP (purity $\geq 99 \%, K$ value $90 \sim 100$, from Sigma-
Fig. 1 a Schematic evolution of the directive orientation of CNTs in laminar fluid flow. The colour represents the velocity gradient, red for high and blue for low. The size and the direction of arrows represent the velocity and flowing direction of the fluid flow, respectively. b Definition of the tilt angle. c SEM photograph of the sample in this wok, proves the alignment of the CNTs using DIW printing process. $\mathbf{d}$ Statistical analysis on the tilt angle. Sample size for the statistical analysis to form the bar graph is 189 (a)

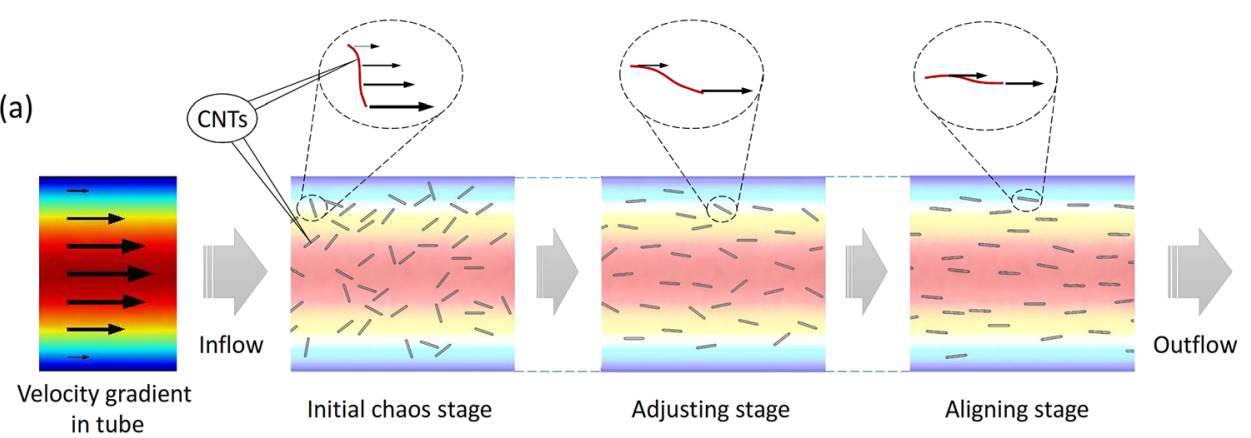

CNTs
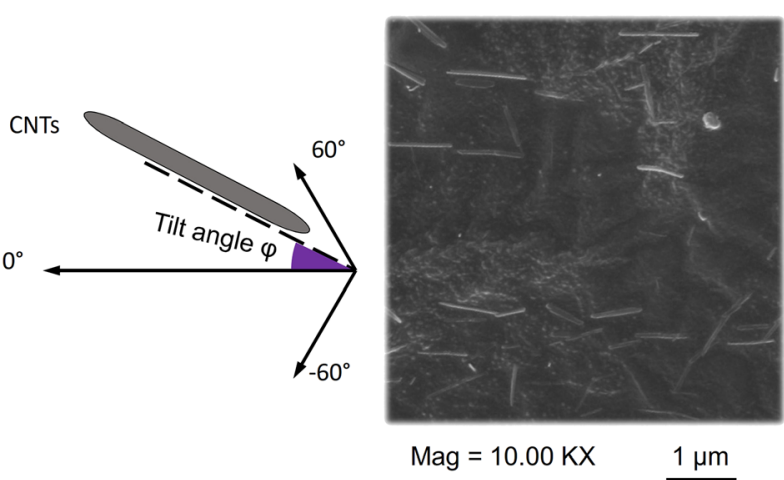

(c)

(d)

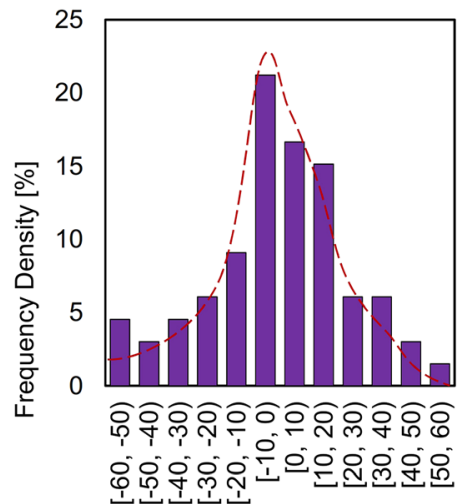

(b)

Tilt angle $\varphi\left[^{\circ}\right]$ 
Aldrich Co. Ltd.) and nanofillers CNTs (Multi-walled carbon nanotubes, purity $\geq 90 \mathrm{wt} \%$, length $1 \sim 3 \mu \mathrm{m}$, from Chengdu Organic Chemicals Co. Ltd.) in a powder form were added into the solution. The dissolved PVP is able to thicken the aqueous solution, thus, facilitating the uniform dispersion of CNTs. After $24 \mathrm{~h}$ of stirring, the photocuring precursor in form of suspension slurry was ready to be loaded in a syringe. The content of CNTs herein is defined using the weight/volume percentage $(\mathrm{w} / \mathrm{v} \%)$, which denotes the mass of CNTs per $100 \mathrm{ml}$ of precursor.

\subsection{Printing process and post-treatments}

The HEMA-based DIW-printing process proposed in this paper is schematically illustrated in Fig. 2. First, the photocuring precursor was prepared and put into a syringe. Ammonium persulfate solution (APS, $10 \mathrm{wt} \%$, purity $98 \%$, from Sigma-Aldrich Co. Ltd.) acting as the initiator was then mixed with the precursor by employing a static mixer to start the printing process (see Fig. 2). The length of the static mixer was determined by FEA simulation to ensure that homogeneous mixture was formed. Simulation results can be found in Fig. S1 in supplementary material. Finally, the hybrid ink was extruded from the nozzle and cured with UV light to form poly(2-hydroxyethyl methacrylate) (PHEMA). Thanks to the shear-induced alignment of CNTs, the printing direction dictates their local orientation. Following the programmable printing routes, samples of the hydrogel nanocomposite with tailored CNT orientations were thus built using the DIW technique. Three representative directions of the CNTs alignments were chosen, namely, the longitudinal direction, the diagonal direction and the transverse direction. The dimensions of the final samples are $40 \mathrm{~mm}$ in length, $10 \mathrm{~mm}$ in width and $0.8 \mathrm{~mm}$ in thickness.
The extrusion rate of the hybrid ink and the velocity of nozzle movement should be well balanced to match the viscosity of the precursor. A rheological curve of the precursor at room temperature is shown in Fig. 2. The viscosity was measured at shear rates from $10^{-3}$ to $10^{3} \mathrm{~s}^{-1}$; it decreased with the increase in the shear rate. In this case, the maximum shear rate of the hybrid ink for the whole pipe system was at the nozzle. For a nonNewtonian fluid flowing within a pipe, the shear rate at the inner wall is given by the Weissenberg-RabinowitschMooney equation $[17,18]$

$\dot{\gamma}=\frac{4 Q}{\pi r^{3}}\left(\frac{3 n+1}{4 n}\right)$

where $\dot{\gamma}$ is the shear rate, $Q$ is the volumetric flow rate inside the pipe, $r$ is the internal pipe radius, and $n$ is the flow index ( $n$ $<1$ in this case, indicating the fluid is shear-thinning). For the optimal printing parameters developed in this work, the shear rate was calculated as $179.26 \mathrm{~s}^{-1}$, which derives the viscosity of the hybrid ink as $3.02 \mathrm{~Pa}$.

Although a high speed of the moving nozzle can improve the manufacturing efficiency, it should be matched with the extrusion rate $[19,20]$. If the extrusion rate is high, excess hybrid ink that cannot solidify in time causes overlaps of the printed traces. On the other hand, a low extrusion rate can cause thin and intermittent printed traces, thereby resulting in discontinuous samples. Based on our experimental trials, the optimal combination of printing parameters used in this work is listed in Table 1.

After the UV curing photopolymerising reactions were completed, the activity of the free radicals, which were released by HEMA monomer, was lost. Stable and crosslinked polymeric chains were formed to hold the embedded CNTs together and froze their orientations. At this stage, the general physical state of the printed sample is hard and sticky,
Fig. 2 Schematic diagram of DIW printing process. The rheological curve of the precursor was measured with a rheometer with two coaxial parallel plates (Ø35 mm, distance $1 \mathrm{~mm}$ ). Angle $\theta=0^{\circ}, 45^{\circ}$ and $90^{\circ}$ defines the longitudinal, diagonal and transverse samples, respectively. Dimensions of the sample are as follows: length $=40 \mathrm{~mm}$, width $=$ $10 \mathrm{~mm}$ and thickness $=0.8 \mathrm{~mm}$
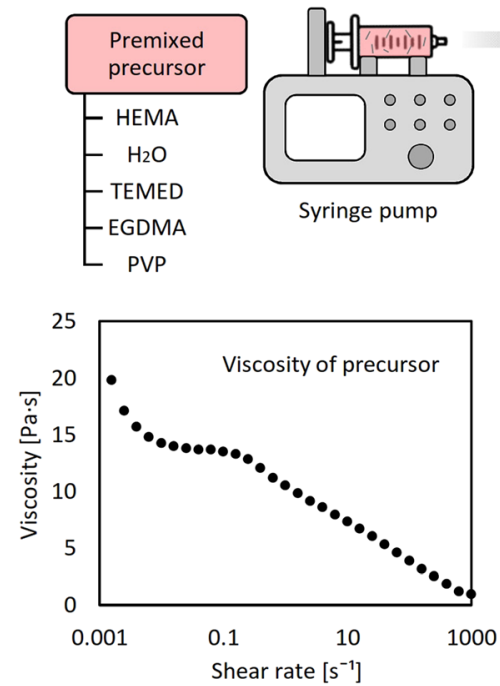
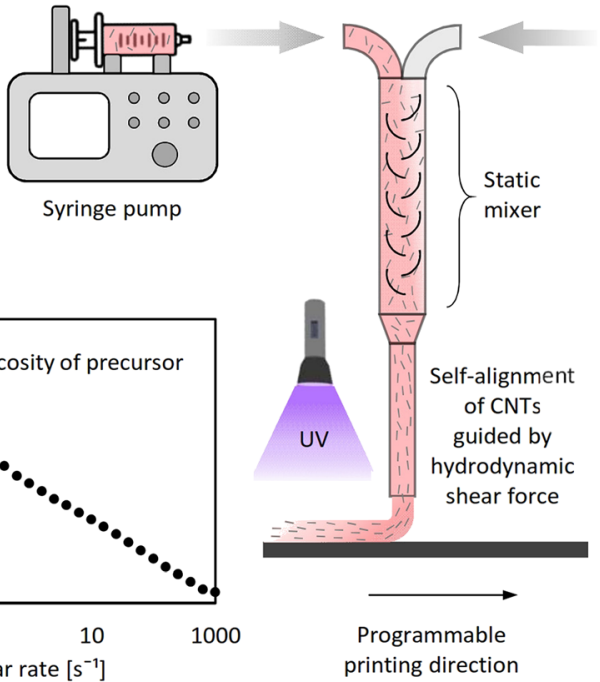
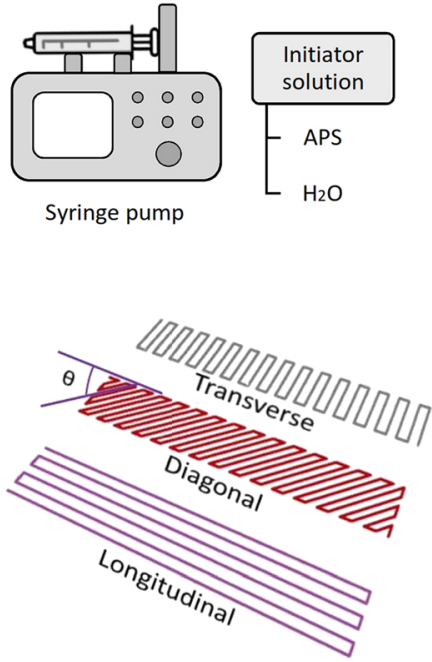
Table 1 Optimal combination of printing parameters

\begin{tabular}{ll}
\hline Printing parameter & Value \\
\hline Nozzle diameter & $0.6 \mathrm{~mm}$ \\
Layer height (distance between exit and substrate) & $0.4 \mathrm{~mm}$ \\
Extrusion rate from the syringe pump (precursor) & $30.41 \mu \mathrm{L} / \mathrm{s}$ \\
Extrusion rate from the syringe pump (initiator) & $3.93 \mu \mathrm{L} / \mathrm{s}$ \\
Printing speed (moving speed of the nozzle) & $40 \mathrm{~mm} / \mathrm{s}$ \\
\hline
\end{tabular}

because the PHEMA polymeric chains are tightly entangled with each other at microscale. To release the residual stresses between the polymer chains and CNTs, swelling of the samples should be carried out. So, the samples were first immersed into a $30 \mathrm{vol} \%$ ethanol aqueous solution for $4 \mathrm{~h}$. Small solvent molecules entering the material pushed the polymeric chains apart, thereby, releasing the residual stresses. The concentration of ethanol was subsequently diluted by repeatedly replacing the soak solution with deionised water. This process not only released the internal stress but also enabled the cleansing of the chemical residuals that remained inside the hydrogel product.

\subsection{Experimental apparatus}

The DIW printing platform used in this work was based on a modified commercial fused deposition (FDM) delta printer (model QQ, Henan FLSUN, China). In brief, its original extruder and feeding system were replaced with the adapted mixing and injecting pipe system, connected with two independent screw-driven syringe pumps (customised model, Xinka electronics, China). To evenly mix the precursor and the initiator, a static mixer with a series of twisted baffles was employed. As the streams moved through the mixer, each set of baffles was able to divide the incoming stream into two. The more baffles in the mixer, the more striations of fluids discharged from the mixer, thus, resulting in an exponential increase in stratification [21]. In this work, the static mixer consisting of five baffles was used, installed inside the tube just before the nozzle.

A uniaxial mechanical testing apparatus (Model UTM6503, SUNS Industrial Testing System Co. Ltd., China) equipped with $200 \mathrm{~N}$ of load cell (Transcell Scale Co. Ltd., USA) was employed to conduct the uniaxial quasistatic stretching and dynamic cyclic loading tests. Two digital multimetres (model VC97, TASI Electronics Co. Ltd., China) were utilised to evaluate the electric conductivity of the hydrogel nanocomposite, based on a four-terminal sensing method. To figure out the electromechanical coupling property of the nanocomposite, e.g. piezoresistivity, an electrochemical workstation (Model CS310H, Wuhan CorrTest Instruments Co. Ltd., China) associated with the SUNS mechanical testing machine was set up. The voltage-current curves were recorded for stretching of the samples with various strain rates.

\section{Results and discussion}

\subsection{Mechanical characteristics}

\subsubsection{Uniaxial quasi-static stretching}

According to our previous research $[22,23]$, there is a high likelihood that the mechanical properties of PHEMA-based nanocomposites are viscoelastic or hyperelastic. For a typical viscoelastic material, the phase difference between the stress input and the strain response leads to a hysteresis, known as dissipated energy [24-26]. Thus, the stress-strain relationship of the material is sensitive to the loading speed or strain rate $[27,28]$. To minimise the impact of the hysteretic resilience on the stress-strain curve, a stretching speed of $5 \mathrm{~mm} / \mathrm{min}$ (corresponding to $0.0021 \mathrm{~s}^{-1}$ in strain rate) was used for the uniaxial tensile test, which was low enough to consider the stretching process as quasistatic loading.

The stress-strain curves of fully swollen PHEMA-CNT hydrogel nanocomposites with varied concentrations of CNTs are demonstrated in Fig. 3a of longitudinal orientation of CNTs with regard to the uniaxial stretching direction. Compared with the pure PHEMA hydrogel, both PHEMACNT- $0.5 \mathrm{w} / \mathrm{v} \%$ and PHEMA-CNT-1.0 w/v\% samples show robust mechanical properties. The elastic modulus of the initial linear region of the stress-strain curve, also known as the Young's modulus, distinctively increased with the increase in the CNT content. It indicates that the content of CNTs positively altered the stiffness of the nanocomposite. It resulted from enhanced interfacial bonds between CNTs and PHEMA matrix or interconnections between CNTs themselves, e.g. $\pi-\pi$ bonding or electrostatic interaction. With the increase in the CNT content, the strength increased from $0.09 \mathrm{MPa}(0 \mathrm{w} / \mathrm{v} \%)$ to $0.12 \mathrm{MPa}(0.5 \mathrm{w} / \mathrm{v} \%)$ and $0.16 \mathrm{MPa}$ $(0.5 \mathrm{w} / \mathrm{v} \%)$, corresponding to the ultimate strain of $0.46,0.36$ and 0.31 , respectively. Therefore, it reveals a tradeoff between the ductility and the load-bearing capacity because the toughness remained unchanged, although the addition of CNTs enhances the stiffness of the material.

The effect of preferential orientation of CNTs is shown in Fig. 3b: the PHEMA-CNT-0.5 w/v\% samples with all orientations (longitudinal, diagonal and transverse) exhibited identical behaviour at small magnitudes of tensile strain (below 0.2). Theoretically, from the rheological point of view, longitudinally oriented nanofillers can be considered as parallel assembling of rod-like nanoparticles in the matrix, which can be described with the Voigt model [29]. While transversely oriented nanofillers can be regarded as a serial assembling 


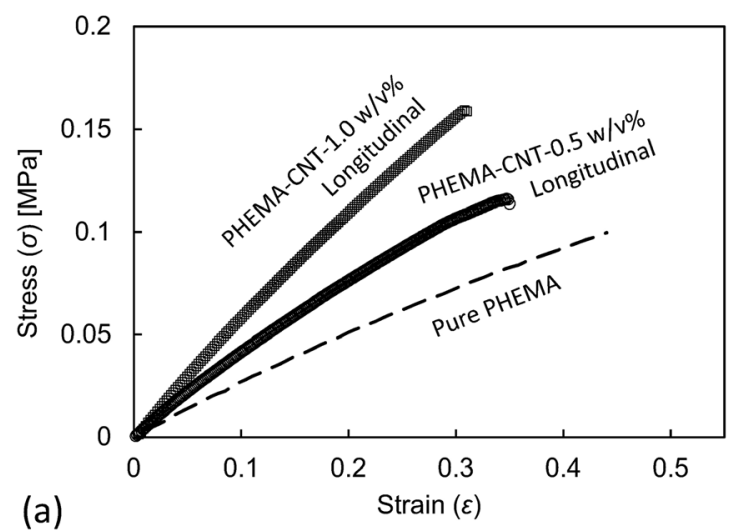

Fig. 3 a Stress-strain curves of PHEMA hydrogel and PHEMA-CNT$0.5 / 1.0 \mathrm{w} / \mathrm{v} \%$ hydrogel nanocomposite samples with longitudinally aligned CNTs. b Stress-strain curves of PHEMA-CNT-0.5 w/v\%

of fillers in the matrix according to the Reuss model [30]. The overall modulus derived by these two models is given by.

Voigt model (applicable for longitudinal alignment) $: E=f E_{f}+(1-f) E_{m}$,

Reuss model (applicable for transverse alignment) $: E=\left(\frac{f}{E_{f}}+\frac{1-f}{E_{m}}\right)^{-1}$,

where $E_{f}$ is the modulus of nanofillers, $E_{m}$ is the modulus of matrix, and $f$ is the volume fraction of the nanofillers. Since identical stress-strain curves were observed at small deformation in Fig. 3b, the CNT aligned directions had an indistinct impact on the modulus that can be attributed to a low volume fraction of CNTs in the nanocomposites, which is calculated as $3.45 \%$ for a PHEMA-CNT- $0.5 \mathrm{w} / \mathrm{v} \%$ sample. As the strain level increased in excess of 0.2 , the hydrogel nanocomposite samples presented varying fracture behaviours. For the PHEMA-CNT- $0.5 \mathrm{w} / \mathrm{v} \%$ nanocomposite, the sample with diagonal orientation of CNTs gave the smallest fracture stress at 0.076 MPa, while the sample with transverse CNTs orientation failed at the stress of $0.092 \mathrm{MPa}$. The sample with the longitudinal orientation of CNTs was the strongest, with the fracture stress of $0.117 \mathrm{MPa}$ at strain of 0.351 . From these experimental observations, apparently, the directional orientation of the CNTs is able to regulate the ultimate strength of the nanocomposite. As well known, ductile material failure is likely to occur in the region with highest von Mises stress. As reported in the literature [31,32], von Mises stress is primarily concentrated and stored at the interface between the CNTs and the matrix, or the entanglement points of polymeric chains. In the case of uniaxial stretching, the principal stress plays a major role in stretching the sample with longitudinal CNTs, while shear stress is responsible for bending of CNTs in the transverse sample or debonding of CNTs from the PHEMA matrix. For the sample with diagonal CNTs,

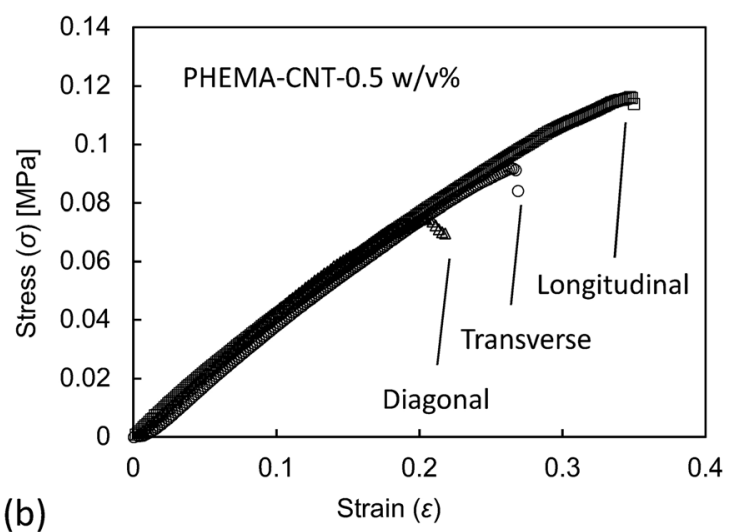

hydrogel nanocomposites for three orientations of CNTs. Each curve is plotted by averaging 3 groups of data. The maximal strain was set as 0.4

comparable principal and shear stresses are applied and concentrated at the CNT entanglements, thereby, attributing to the maximum von Mises stress and resulting in the smallest fracture stress.

\subsubsection{Dynamic cyclic loading}

To quantitatively evaluate the hysteresis of viscoelastic hydrogel nanocomposites, cyclic loading-unloading tests were conducted. Twenty cycles of loading and unloading with two speeds $-50 \mathrm{~mm} / \mathrm{min}$ and $100 \mathrm{~mm} / \mathrm{min}$ - were implemented on the PHEMA-CNT- $0.5 \mathrm{v} / \mathrm{w} \%$ samples with different CNT orientations. According to the literature [33,34], a hysteretic loop formed by the loading and unloading stress-strain curves can be observed in viscoelastic materials. The size of the loop represents the energy dissipated during the cyclic deformation. The degree of viscoelasticity $(\eta)$ is defined as the ratio of the unloading area (the area under the unloading path) to the loading area (the area under the loading path) [24]. Thus, the more energy dissipated during the cyclic loading, the larger hysteretic loop can be obtained; consequently, the ratio $\eta$ is farther from the value 1, indicating a larger degree of viscoelasticity. Particularly, if the ratio $\eta$ equals to 1 , the mechanical property of the material can be categorised as hyperelasticity because there is no energy dissipation during the cyclic loading.

Evolution of stress-strain curves in dynamic cyclic loading is presented in Fig. 4. No offset was observed for the cycle. The small triangular overshoots (near the minimal and maximal strains) were caused by the inertial torque of a servo motor when reversing its rotating speed. These triangular overshoots have been manually neglected in calculating the size of hysteretic loops. In general, the loading-unloading loops coincided with each other, indicating that PHEMA-CNT hydrogel nanocomposites had stable and fully recoverable mechanical parameters under dynamic cyclic loading. For the same 

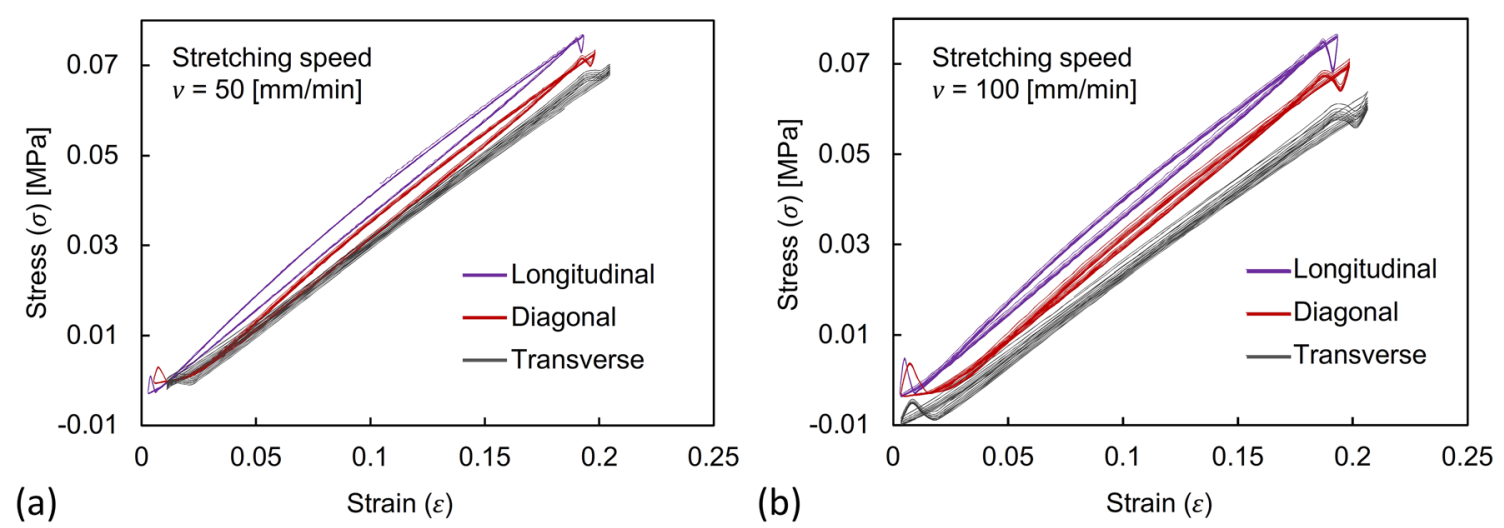

Fig. 4 Stress-strain curves from dynamic cyclic loading at speed of $50 \mathrm{~mm} / \mathrm{min}$ (a) and $100 \mathrm{~mm} / \mathrm{min}$ (b) for PHEMA-CNT-0.5 v/w\% sample with longitudinal, diagonal and transverse CNTs. The maximal strain was set as 0.2

loading speed, the largest size of the hysteretic loop was deserved for the longitudinal sample. The values $\eta$ were calculated as 0.923 and 0.944 , for Fig. $4 \mathrm{a}$ and $\mathrm{b}$ respectively. As the orientation angle $\theta$ increased from 0 to $90^{\circ}$, the size of hysteretic loop decreased for both loading speeds. The respective curves for the sample with transverse aligned CNTs exhibited the minimum size of hysteretic loops, regarding in the ratio $\eta$ of 0.997 (for Fig. 4a) and 0.998 (for Fig. 4b). In terms of the size of the hysteretic loop, the samples with diagonal reinforcement fall between the "longitudinal" and "transverse" groups. The variation of the ratio $\eta$ for pure PHEMA was confined as $0.97 \sim 0.99$, because of its hyperelasticity [35]. Therefore, comparing the values of ratio $\eta$ for the pure PHEMA and the hydrogel nanocomposite samples under loading speed of 50 and $100 \mathrm{~mm} / \mathrm{min}$ shown in Fig. $4 \mathrm{a}$ and b, two main findings can be outlined: (1) the loading speed has a limited impact on the size of hysteretic loop; (2) viscoelasticity of the hydrogel nanocomposite originates from the presence of CNTs, which can be altered by their orientation.

Hence, as a brief summary of the mechanical characterisation of the PHEMA-CNT hydrogel nanocomposite, anisotropic mechanical behaviour experimentally established is a result of the orientation of CNTs that can regulate the strength and the degree of viscoelasticity of the material.

\subsection{Electromechanical coupling}

\subsubsection{Electric resistivity}

Electric-resistivity tests were conducted based on the fourterminal sensing method using two digital multimetres (model VC97, TASI Electronics Co. Ltd., China), aiming to gain quantitative understanding on the impact of orientation of CNTs on electric conductivity. Four-terminal sensing, also known as 4-point probes method or Kelvin sensing, is an accurate resistance or impedance measuring technique [36]. In a simple two-point measurement, the measured resistance includes not only that of the test sample but also that of the test probes themselves. In four-terminal sensing, the separation of current and voltage electrodes eliminates the contribution of resistance of the current leads to the measured voltage drop, thereby, leading to an accurate resistance value for the test sample. Four-terminal sensing was adopted in this work because it is suitable for measuring the sheet resistance of films [37].

The experimental setup for the electric resistivity tests is schematically illustrated in Fig. 5a. A sheet-like printed hydrogel nanocomposite with anisotropic CNTs alignment (thickness $t=0.86 \mathrm{~mm}$ ) was placed on an insulating substrate (glass in this study). Four electrodes were arranged in a line. The current was applied to the surface at position 1 leaving in position 2. Similarly, the voltage was charged between positions 3 and 4 . The distances between 1 and 3 and 2 and 4 were fixed as $d_{13}=d_{24}=4 \mathrm{~mm}$ in this case. During the test, four variables were recorded including the distance between positions 3 and $4\left(d_{34}\right)$, the angle $(\theta)$, the voltage $(U)$ and the current $(I)$, read from the digital multimetres. The experimental results in terms of the calculated resistance values are depicted in Fig. 5b. As the overall trend, the resistance calculated for all orientations slightly increased with the distance between the electrodes, when $d_{34}$ ascended from 1 to $5 \mathrm{~cm}$. The largest (smallest) resistance was always identified for the diagonal (longitudinal) orientation for each value of $d_{34}$. This difference caused by the CNT orientation became inconspicuous with the increase in $d_{34}$. Thus, the printed hydrogel nanocomposite demonstrated anisotropic electric conductive properties, related to the orientation of CNTs. To gain further information about the contribution of orientation of CNTs to the anisotropic properties of the hydrogel nanocomposites, coupling of mechanical and electrical behaviours could provide fundamental proofs and quantitative explanations.

\subsubsection{Piezoresistivity}

To investigate the coupling effect of mechanical and electrical behaviours of the PHEMA-CNT hydrogel nanocomposites, 
Fig. 5 a Schematic diagram of resistivity test setup with fourterminal sensing. b Experimental results of resistivity tests in terms of resistance values (the error bars represent the standard error with $n=5)$

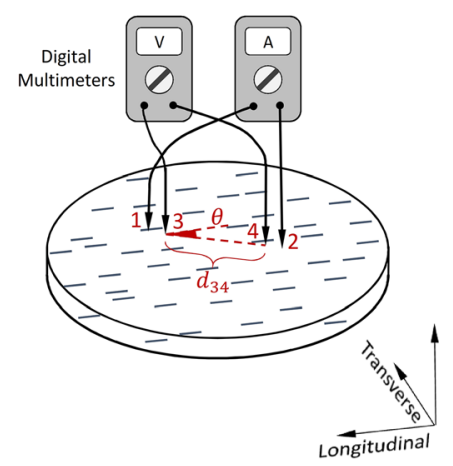

(a)

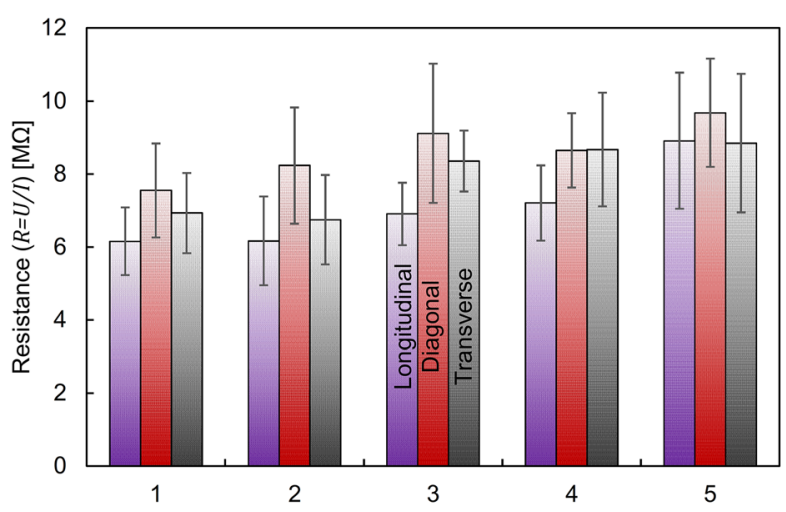

(b)

Distance $\left(d_{34}\right)[\mathrm{cm}]$
$50 \mathrm{~mm} / \mathrm{min}$ (negative speed denotes the reversed direction). The unloading process was terminated when the original length of the sample was reached, thereby leading to stage 6 - static state. The corresponding electrical recordings in terms of the voltage-current characteristic are depicted in Fig. 6a, with the line segments clearly signifying the 6 stages of mechanical loading. The slope of each segment (detailed values can be found in Table S1 in Supplementary Materials) indicates the resistance of the sample under the corresponding loading condition. With the increase of the responsive current from 0 to $2 \mu \mathrm{A}$, the linear voltage-current relationship demonstrates that the resistance of the sample did not vary with the external stress if the strain rate was constant. For the responsive current in excess of $2 \mu \mathrm{A}$, the change of the slopes is apparent, especially for the transition from stage 2 to stage 3. A drastic decrease of the slope can be found at the starting point of the unloading stage, corresponding to the responsive current of $3.71 \mu \mathrm{A}$ in Fig. 6a. When the sample reached its original length at the end of the unloading stage, mechanical stress was fully removed.

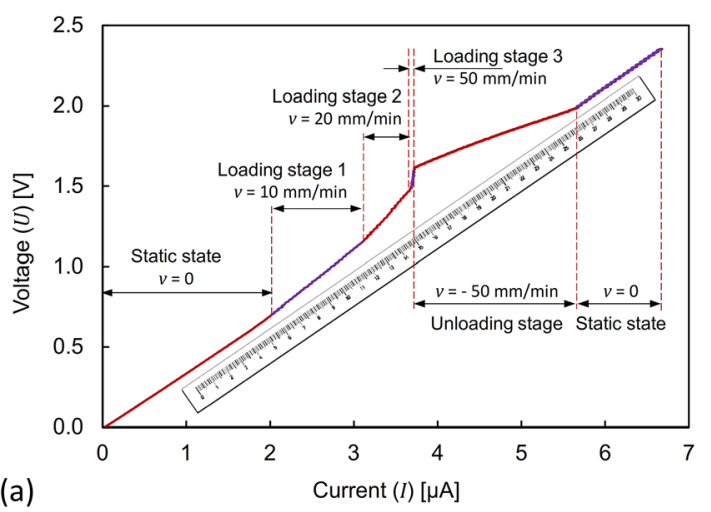

Fig. 6 a Voltage-current characteristic of PHEMA-CNT- $0.5 \mathrm{v} / \mathrm{w} \%$ longitudinal sample for one cycle of uniaxial loading and unloading, with stretching speed $v=0,10,20,50,-50 \mathrm{~mm} / \mathrm{min}$. b Influence of orientation of CNTs on resistivity of sample for different stretching speeds. The

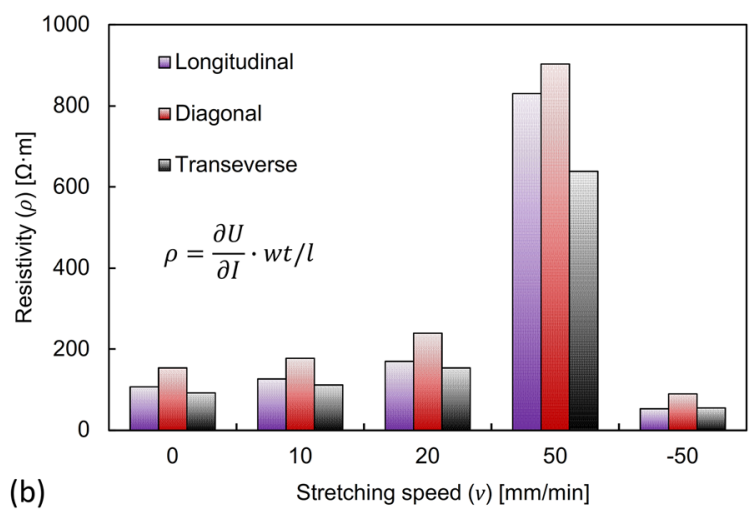

resistivity was derived based on the slope of the voltage-current curve $(\partial U / \partial I)$ and the effective dimensions of the sample (width $w$, thickness $t$, length $l$ ). Additional data for samples with different orientations and content of CNTs can be found in Supplementary Materials 
As expected, the slope for the final static stage ( $I=$ $5.73 \mu \mathrm{A} \sim$ ultimate) were identical to that of the initial (static) stage $(I=0 \sim 2 \mu \mathrm{A})$. It confidently proves that the speed of applying loads had a significant impact on the voltage-current curves of the PHEMA-CNT hydrogel nanocomposites.

Unlike a normal piezoresistive effect with the change in electrical resistance resulting from the applied external force, the obtained experimental results demonstrated that the electrical resistance of the PHEMA-CNT hydrogel nanocomposite was sensitive to the loading speed instead. Thus, it can be categorised as strain-rate-dependent piezoresistive material. The level of resistivity of samples with different orientations of CNTs (i.e. longitudinal, diagonal, transverse), is compared in Fig. 6b, for various loading speeds. For any loading speed, the resistivity of the nanocomposite with diagonal reinforcement was the largest. It increased from 178 to $917 \Omega \cdot \mathrm{m}$ with the loading speed 0 to $50 \mathrm{~mm} / \mathrm{min}$ respectively. Distinguishable differences between the resistivity values of the transverse and longitudinal samples were observed, especially at the loading speed of $50 \mathrm{~mm} / \mathrm{min}$ (up to some $200 \Omega \cdot \mathrm{m}$ ). Compared with the resistivity of static and loading stages, that of the unloading stage was the lowest for the samples with all three CNT orientations.

According to the literature [38, 39], piezoresistivity arises from the deformation of the energy bands as a result of applied stress. Therefore, the piezoresistivity of the hydrogel nanocomposite can be explained using the change of the energy bandgap in the CNT networks due to external stress or applied strain. The relationship between the energy bandgap $\left(E_{g a p}\right)$ and strain $(\varepsilon)$ caused by the external stress can be given by

$\frac{\partial E_{\text {gap }}}{\partial \varepsilon}=\operatorname{sgn}(2 p+1) \cdot 3 t_{0}(1+\nu) \cos (3 \varphi)$,

where $t_{0}=2.74 \mathrm{eV}$ is the constant of the carbon nanotubes, $\varphi$ is the chiral angle, $\nu$ is the Poisson's ratio, $\operatorname{sgn}(2 p+1)$ is the signum function that is used to determine the strain direction, $p=0,1$ or -1 . This understanding provides a theoretical explanation to the dependence of the resistivity on the change in the strain rate: the large strain rate corresponding to a high speed of loading/unloading of the sample induces drastic changes of the energy bandgap, reducing the transmission probability of electrons from a valence band to a conduction band, consequently leading to a large resistivity.

To assess the dependence of the resistivity on the strain rate, the evolution of the piezoresistive coefficient (normally a $6 \times 6$ matrix [40]) was determined. In practical applications, the equation for calculating the piezoresistive coefficient of a thin strip under uniaxial stress can be simplified as a bidirectional model, in terms of the stress components which are parallel and perpendicular to the loading direction. The general model can be expressed as

$\frac{\Delta \rho}{\rho}=\sum \pi_{i j} \cdot \sigma_{i j}$, where $\Delta \rho$ and $\rho$ are the change in resistivity and the original resistivity (e.g. for the static state) respectively, $\sigma_{i j}$ and $\pi_{i j}$ are the tensors of stress and piezoresistive coefficients, respectively. Due to the weak dependence on shape and aspect ratio of cross-sectional area, the stress components can be reduced to the combination of the items that are parallel and transverse to the loading direction $(0<n<1)$, providing an approximate model for assessing the sensitivity of the piezoresistivity under uniaxial stress for thin strip samples:

$\frac{\Delta \rho}{\rho} \cong n \cdot \pi_{\text {parallel }} \cdot \sigma_{\text {parallel }}+(1-n) \cdot \pi_{\text {transverse }} \cdot \sigma_{\text {transverse }}, 0<n<1$.

Evolution of the piezoresistive coefficient, which is parallel to the loading direction, is illustrated in Fig. 7. Four segments of curves can be identified corresponding to the loading and unloading stages. For all the magnitudes of the loading/ unloading speeds, the curves for longitudinal alignments of CNTs demonstrate a unique behaviour: they are located above those for other two orientations. This indicates that the hydrogel nanocomposite with longitudinally oriented CNTs is highly sensitive to the strain change. Additionally, for some loading stages, it can be found that curves for samples with diagonal and transverse CNTs, (i.e. red and grey curves) coincide, e.g. at $10 \mathrm{~mm} / \mathrm{min}$ loading speed. Distinct behaviours of the samples with various orientations of CNTs were found at the unloading stage. It is consistent with the experimental results shown in Fig. 6b: the weak dependence of the voltage-current characteristics on the strain rate during relaxation due to a low band energy, thus, revealing the low level of resistivity.

The values of the calculated piezoresistive coefficient of the PHEMA-CNT hydrogel nanocomposite in this work were in the range from 10.28 to $851.64 \mathrm{MPa}^{-1}$. They were significantly greater than those for (1) CNTs adhered to pressurised membranes, i.e. $0.8 \sim 1.7 \mathrm{MPa}^{-1}$ [41], and (2) SU-8/SWCNT composite, i.e. $0.004 \sim 0.008 \mathrm{MPa}^{-1}$ [42]. This can be

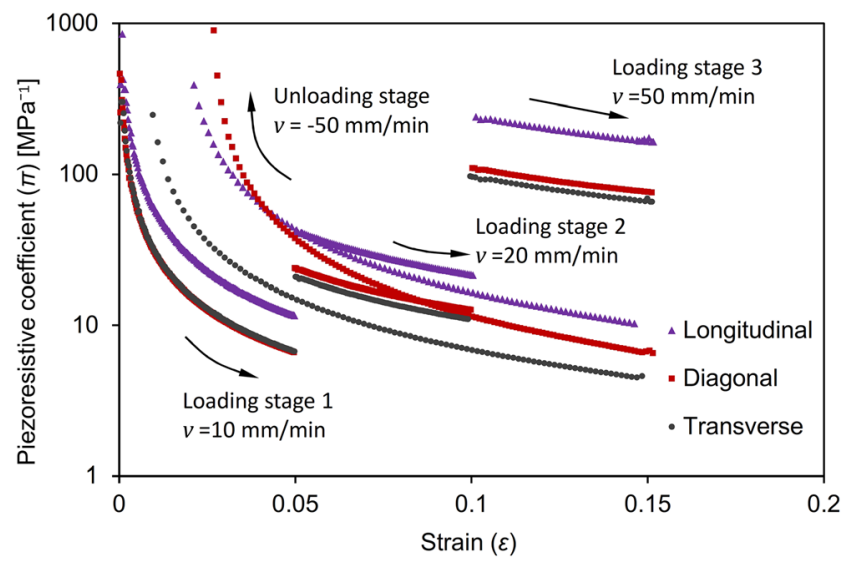

Fig. 7 Correlations between piezoresistive coefficients and strain under uniaxial loading and unloading with different speeds, for PHEMA-CNT$0.5 \mathrm{v} / \mathrm{w} \%$ longitudinal, diagonal and transverse orientations of CNTs 
explained by the fact that the PHEMA-CNT hydrogel nanocomposite is a soft piezoresistive material with high ductility: their responsive strain can reach up to $35 \%$. Thanks to these mechanical and electrical properties, the PHEMA-CNT hydrogel nanocomposite can be considered as potential and effective candidate material that integrates the functions of cushioning and vibration detecting in aqueous environment.

\section{Conclusions}

The novelty of this work lies in that a PHEMA-based hydrogel nanocomposite with anisotropic mechanical and electrical properties is fabricated with DIW printing of CNT-suspended precursor. During the printing process, hydrodynamic shear resulted in alignment of CNTs as the viscous suspension flowed through the deposition nozzle, thus, forming hydrogel product with uniform orientation of CNTs after polymerisation. Results from the quasistatic stretching tests demonstrated that (1) the content of the embedded CNTs enhanced the stiffness of the hydrogel nanocomposite, but with unchanged toughness, and (2) the orientation of the CNTs was able to alter the strength of the hydrogel nanocomposite. Specifically, the samples with diagonal orientation of CNTs exhibited the lowest fracture stress, which can be attributed to the stress concentration on the CNTs entanglement at microscale. Dynamic cyclic loading experimentally proved the viscoelasticity of the studied hydrogel nanocomposites.

Electromechanical properties were evaluated comprehensively by coupling the uniaxial cyclic stretching and voltagecurrent characterisation based on four-terminal sensing. Experimental results demonstrated that orientation of CNTs had an impact on the resistance and resistivity of the nanocomposite, thus, causing their anisotropic electric conductive properties. Moreover, it was demonstrated that the resistivity of the PHEMA-CNT hydrogel depended strongly on the loading speed, (i.e. strain rate), which can be explained by the theory of energy bandgap of CNT networks. Quantitative assessment of sensitivity of the piezoresistivity and the piezoresistive coefficient of the nanocomposites was conducted. Thanks to their fine-tuning nanostructure, which is attributed to the programmable fabrication process, the PHEMACNT hydrogel nanocomposites developed in this work have a great potential as candidates for conductive energy absorbers in aqueous environment, such as nucleus pulposus for biomimetic robots.

Acknowledgements The authors wish to thank the Hubei Digital Manufacturing Key Laboratory at the WUT for performing characterisation of various samples.

Funding information The original research was supported by the National Natural Science Foundation of China $(51703176,91848102)$ and the Fundamental Research Funds for the Central Universities (WUT2018IVB006).

\section{Compliance with ethical standards}

Conflict of interest The authors declare that they have no conflict of interest.

\section{References}

1. Jiang P, Yan C, Guo Y, Zhang X, Cai M, Jia X, Wang X, Zhou F (2019) Direct ink writing with high-strength and swelling-resistant biocompatible physically crosslinked hydrogels. Biomater Sci 7: $1805-1814$

2. Lee KY, Mooney DJ (2001) Hydrogels for tissue engineering. Chem Rev 101(7):1869-1880

3. Huang TQ, Qu X, Liu J, Chen S (2014) 3D printing of biomimetic microstructures for cancer cell migration. Biomed Microdevices 16: $127-132$

4. Whitely M, Cereceres S, Dhavalikar P, Salhadar K, Wilems T, Smith B, Mikos A, Cosgriff-Hernandez E (2018) Improved in situ seeding of 3D printed scaffolds using cell-releasing hydrogels. Biomater 185:194-204

5. Johnson R, Halder G (2014) The two faces of hippo: targeting the hippo pathway for regenerative medicine and cancer treatment. Nat Rev Drug Discov 13:63-79

6. Li Y, Wang S, Huang R, Huang Z, Hu B, Zheng W, Yang G, Jiang X (2015) Evaluation of the effect of the structure of bacterial cellulose on full thickness skin wound repair on a microfluidic chip. Biomacromol. 16(3):780-789

7. Odent J, Wallin TJ, Pan W, Kruemplestaedter K, Shepherd RF, Giannelis EP (2017) Highly elastic, transparent, and conductive 3D-printed ionic composite hydrogels. Adv Funct Mater 27: 1701807

8. Chen Y, Han P, Vandi L, Dehghan-Manshadi A, Humphry J, Kent D, Stefani I, Lee P, Heitzmann M, Cooper-White J, Dargusch M (2019) A biocompatible thermoset polymer binder for direct ink writing of porous titanium scaffolds for bone tissue engineering. Mater Sci Eng C 95:160-165

9. Mulakkal MC, Trask RS, Ting VP, Seddon AM (2018) Responsive cellulose-hydrogel composite ink for 4D printing. Mater Design 160:108-118

10. Grigoryan B, Paulsen SJ, Corbett DC, Sazer DW, Fortin CL, Zaita AJ, Greenfield PT, Calafat NJ, Gounley JP, Ta AH, Johansson F, Randles A, Rosenkrantz JE, Louis-Rosenberg JD, Galie PA, Stevens KR, Miller JS (2019) Multivascular networks and functional intravascular topologies within biocompatible hydrogels. Science 364(6439):458-464

11. Murphy SV, Atala A (2014) 3D bioprinting of tissues and organs. Nat Biotechnol 32(8):773-785

12. Ji Z, Yan C, Yu B, Zhang X, Cai M, Jia X, Wang X, Zhou F (2019) 3D printing of hydrogel architectures with complex and controllable shape deformation. Adv Mater Technol 2019:1800713

13. Wang J, Lu T, Yang M, Sun D, Xia Y, Wang T (2019) Hydrogel 3D printing with the capacitor edge effect. Sci Adv 5(3):eaau8769. https://doi.org/10.1126/sciadv.aau8769

14. Cai J, Li X, Ma L, Jiang Y, Zhang D (2019) Facile large-scale alignment and assembly of conductive micro/nano particles by combining both flow shear and electrostatic interaction. Compos Sci Technol 171:199-205

15. Compton BG, Lewis JA (2014) 3D-printing of lightweight cellular composites. Adv Mater 26:5930-5935 
16. Gladman AS, Matsumoto EA, Nuzzo RG, Mahadevan L, Lewis JA (2016) Biomimetic 4D printing. Nat Mater 15:413-418

17. Darby R (2001) Chemical engineering fluid mechanics, 2nd edn. CRC Press

18. Dorf RC (2004) The engineering handbook, 2nd edn. CRC Press, Florida

19. Hong S, Sanchez C, Du H, Kim N (2015) Fabrication of 3D printed metal structures by use of high-viscosity $\mathrm{Cu}$ paste and a screw extruder. J Electron Mater 44:836-841

20. Ren X, Shao H, Lin T, Zheng H (2016) 3D gel-printing-an additive manufacturing method for producing complex shape parts. Mater Des 101:80-87

21. Paul EL (2004) Handbook of industrial mixing-science and practice. John Wiley \& Sons, Hoboken NJ

22. Zhao W, Shi Z, Chen X, Yang G, Lenardi C, Liu C (2015) Microstructural and mechanical characteristics of PHEMA-based nanofibre-reinforced hydrogel under compression. Compos B Eng 76:292-299

23. Zhao W, Li X, Gao S, Feng Y, Huang J (2017) Understanding mechanical characteristics of cellulose nanocrystals reinforced PHEMA nanocomposite hydrogel: in aqueous cyclic test. Cellulose 24(5):2095-2110

24. Gao X, Shi Z, Lau A, Liu C, Yang G, Silberschmidt VV (2016) Effect of microstructure on anomalous strain-rate-dependent behaviour of bacterial cellulose hydrogel. Mater Sci Eng C 62:130-136

25. Cao C, Gao X, Conn AT (2019) A compliantly coupled dielectric elastomer actuator using magnetic repulsion. Appl Phys Lett 114: 011904

26. Gao X, Shi Z, Liu C, Yang G, Sevostianov I, Silberschmidt VV (2015) Inelastic behaviour of bacterial cellulose hydrogel: in aqua cyclic tests. Poly Testing 44:82-92

27. Gao X, Shi Z, Kuśmierczyk P, Liu C, Yang G, Sevostianov I, Silberschmidt VV (2016) Time-dependent rheological behaviour of bacterial cellulose hydrogel. Mater Sci Eng C 58:153-159

28. Gao X, Kuśmierczyk P, Shi Z, Liu C, Yang G, Sevostianov I, Silberschmidt VV (2016) Through-thickness stress relaxation in bacterial cellulose hydrogel. J Mech Behav Biomed Mater 59:9098

29. Voigt W (1889) Ueber die Beziehung zwischen den beiden Elasticitatsconstanten isotroper Korper. Ann Phys 274:573-587
30. Reuss A (1929) Berechnung der Fließgrenze von Mischkristallen auf Grund der Plastizitatsbedingung fur Einkristalle. J Appl Math Mech 9:49-58

31. Gan L, Dong M, Han Y, Xiao Y, Yang L, Huang J (2018) Connection-improved conductive network of carbon nanotubes in a rubber cross-link network. ACS Appl Mater Interfaces 10(21): 18213-18219

32. Li Y, Stier B, Bednarcyk B, Simon JW, Reese S (2016) The effect of fiber misalignment on the homogenized properties of unidirectional fiber reinforced composites. Mech Mater 92:261-274

33. Shi Z, Zhao W, Li S, Yang G (2017) Self-powered hydrogel induced by ion transport. Nanoscale 9:17080

34. Yamamoto T, Minato T (2007) Theory of energy dissipation in a viscoelastic body under time-dependent stress. Adv Space Res 39(3):472-476

35. Zhao W, Lenardi C, Webb P, Liu C, Santaniello T, Gassa FA (2013) Methodology to analyse and simulate mechanical characteristics of poly (2-hydroxyethyl methacrylate) hydrogel. Polym Int 62:1059 1067

36. Valdes LB (1954) Resistivity measurements on germanium for transistors. Proc IRE 42:420

37. Chandra H, Allen SW, Oberloier SW, Bihari N, Gwamuri J, Pearce JM (2017) Open-source automated mapping four-point probe. Materials 10(2):110

38. Yang N, Chen X, Ren T, Zhang P, Yang D (2015) Carbon nanotube based biosensors. Sensor Actuat B-Chem 207:690-715

39. Zhao W, Shi Z, Hu S, Yang G, Tian H (2018) Understanding piezoelectric characteristics of PHEMA-based hydrogel nanocomposites as soft self-powered electronics. Adv Compos Hybrid Mat 1: $320-331$

40. Kanda Y (1982) A graphical representation of the piezoresistance coefficients in silicon. IEEE Trans Electron Devices 29(1):64-70

41. Grow RJ, Wang Q, Cao J, Wang D, Dai H (2005) Piezoresistance of carbon nanotubes on deformable thin-film membranes. Appl Phys Lett 86:093104

42. Barlian AA, Park WT, Mallon JR, Rastegar AJ, Pruitt BL (2009) Review: semiconductor piezoresistance for microsystems. Proc IEEE 97(3):513-552 